\title{
From Nodeless Clouds and Vortices to Gray Ring Solitons and Symmetry-Broken States in Two-Dimensional Polariton Condensates
}

\author{
A.S. Rodrigues, ${ }^{1}$ P.G. Kevrekidis,${ }^{2}$ R. Carretero-González, ${ }^{3}$ J. Cuevas,${ }^{4}$ D.J. Frantzeskakis,${ }^{5}$ and F. Palmero ${ }^{6}$ \\ ${ }^{1}$ Departamento de Física/CFP, Faculdade de Ciências, \\ Universidade do Porto, R. Campo Alegre, 687 - 4169-007 Porto, Portugal \\ ${ }^{2}$ Department of Mathematics and Statistics, University of Massachusetts, Amherst MA 01003-4515, USA \\ ${ }^{3}$ Nonlinear Dynamical Systems Group, ${ }^{*}$ Department of Mathematics and Statistics, \\ and Computational Science Research Center, San Diego State University, San Diego CA, 92182-7720, USA \\ ${ }^{4}$ Grupo de Física No Lineal. Departamento de Física Aplicada I. Escuela Politécnica Superior, \\ Universidad de Sevilla, C/ Virgen de África, 7, 41011-Sevilla, Spain \\ ${ }^{5}$ Department of Physics, University of Athens, Panepistimiopolis, Zografos, Athens 157 84, Greece \\ ${ }^{6}$ Grupo de Física No Lineal. Departamento de Física Aplicada I. Escuela Técnica Superior de Ingeniería Informática, \\ Universidad de Sevilla, Avda. Reina Mercedes, s/n, 41012-Sevilla, Spain
}

(Dated: January 29, 2014)

\begin{abstract}
We consider the existence, stability and dynamics of the nodeless state and fundamental nonlinear excitations, such as vortices, for a quasi-two-dimensional polariton condensate in the presence of pumping and nonlinear damping. We find a series of interesting features that can be directly contrasted to the case of the typically energy-conserving ultracold alkali-atom Bose-Einstein condensates (BECs). For sizeable parameter ranges, in line with earlier findings, the nodeless state becomes unstable towards the formation of stable nonlinear single or multi-vortex excitations. The potential instability of the single vortex is also examined and is found to possess similar characteristics to those of the nodeless cloud. We also report that, contrary to what is known, e.g., for the atomic BEC case, stable stationary gray ring solitons (that can be thought of as radial forms of Nozaki-Bekki holes) can be found for polariton condensates in suitable parametric regimes. In other regimes, however, these may also suffer symmetry breaking instabilities. The dynamical, patternforming implications of the above instabilities are explored through direct numerical simulations and, in turn, give rise to waveforms with triangular or quadrupolar symmetry.
\end{abstract}

\section{INTRODUCTION}

One of the most rapidly developing branches of studies in the physics of Bose-Einstein condensation is that of exciton-polariton condensates in semiconductor microcavities. Only a few years since their experimental realization ${ }^{1-4}$, exciton-polariton Bose-Einstein condensates (BECs) have become a prototypical system for studies at the interface of non-equilibrium physics and nonlinear dynamics. More specifically, the radiative lifetime of the polaritons provides a short relaxation time scale in the system of the order of $1-10 \mathrm{ps}^{5}$. At the same time, the light mass of these quasi-particles provides them with a considerably higher condensation temperature. Moreover, the photonic component of the system only allows for a short lifetime and no thermalization. Instead, the exciton-polariton system produces a genuinely nonequilibrium condensate, requiring the external pumping of an excitonic reservoir, which in turn balances the polariton loss 5.6 . This "open" nature of the system, featuring gain and loss, is then responsible for its rich pattern forming capabilities that have been recently summarized, e.g., in Refs 7 .

Our interest in the prototypical two-dimensional setting of the system will be precisely at the level of the in-

*URL: http://nlds.sdsu.edu terplay of the intrinsic nonlinearity due to inter-particle interactions and the gain/loss nature of the system. This interplay has led to a wide variety of remarkable observations (and theoretical explorations) including, but certainly not limited to, features such as flow without scattering (analogue of the flow without friction) ${ }^{10}$, the existence of vortices 11 (see also Ref. 12 for vortex dipole dynamics and Ref. 13 for observations thereof), persistent currents as well as higher charge vortices ${ }^{14}$, collective dynamics ${ }^{15}$, solitary wave structures such as bright ${ }^{16}$, $\operatorname{dark}^{17}$ and gap $\frac{18}{}$ solitons, and even remarkable applications such as spin switches 19 and light emitting diodes ${ }^{20}$ operating even near room temperatures.

The approach that has been used most commonly in theoretical studies of exciton-polariton BECs relies on the analysis of two coupled evolution equations for the polaritons and the exciton reservoir which enables their production. In particular, the relevant model assumes the form of two coupled complex Ginzburg-Landau (cGL) equations describing the evolution of exciton and photon wavefunctions $21-23$. However, an alternative that has been proposed ${ }^{9,24-26}$ in the case of incoherent and/or far blue-detuned laser pumping (see, e.g., Ref. 9 and references therein) suggests that a single cGL equation for the macroscopically occupied polariton state may be used instead; such a model yields results consistent with experimental observations 27 (see also Refs. 8, 9).

In what follows, we will consider the case of incoherent pumping and use in our study a single cGL equation. 
Our aim is to analyze in detail some of the fundamental states of the two-dimensional system. In particular, in earlier works these states have been chiefly obtained as attractors of the relevant gain/loss dynamics, revealing the pattern forming complexity that emerges spontaneously in the system. Here, our aim is not only to revisit fundamental states (such as the nodeless cloud or the single vortex) and explore their parametric dependence by developing two-parameter bifurcation diagrams (in parameters such as the gain strength and its spot radius); it is instead to provide a detailed view towards the stability of these states unveiling their spectral properties and the somewhat unusual nature of their instabilities. In addition to these more standard states, we will also consider states that, to the best of our knowledge, have not been previously presented in the context of polariton condensates, although they have been discussed for atomic condensates 28 . A principal example of this form is the so-called ring dark soliton (RDS) which, remarkably, although never stable in the context of atomic BECs ${ }^{29-31}$, can in fact be shown to be stable in suitable (gain) parametric regimes here. This is, effectively, a potentially stable radial form of a Nozaki-Bekki hole ${ }^{32}$ that was previously explored in cGL $\operatorname{contexts}^{33}$, yet was not found to be stable in these settings; instead, it was found there to potentially initiate a form of spiral wave turbulence. Importantly, this ring soliton structure was observed in polariton superfluids in a very recent experiment 34 . We also reveal the symmetry-breaking instabilities of this ring soliton structure and unveil a series of solutions without radial symmetry that may spontaneously emerge as a result of such instabilities. Among them, we highlight the potential for states with triangular or square/rectangular symmetry, whose parametric dependence (as stationary states) we also explore. Finally, for all relevant states, we offer a number of direct numerical simulations that yield insight towards the manifestation of the instabilities and the spontaneous emergence of patterns such as vortex lattices, but also of non-vortical patterns without radial symmetry. We should also note in passing here that a similar study focusing, however, predominantly on the existence properties of some of the solutions considered here (rather than on their stability, which is the principal emphasis herein), and chiefly considering the case without a parabolic trap, was recently published 35 .

Our exposition is structured as follows. In Sec. II we offer the theoretical setup and techniques that will be used. In Sec. III, we present the numerical results in two subsections: the first one provides the bifurcation structure and parametric continuations/stability analysis of the relevant solutions (initially this is done for the nodeless cloud and single vortex, and subsequently for the ring and related symmetry broken states); the second one examines the results of direct numerical simulations results. Finally, in Sec.IV] we summarize our findings, as well as mention some interesting directions for potential future studies.

\section{MODEL SETUP}

As indicated above, we will consider the complex Ginzburg-Landau model developed in Refs. 24 26 (see also Ref. 9 and references therein):

$$
i \partial_{t} \psi=\left\{-\nabla_{\perp}^{2}+r^{2}+|\psi|^{2}+i\left[\left(\chi(r)-\sigma|\psi|^{2}\right)\right]\right\} \psi,
$$

where $\psi$ denotes the polariton wavefunction trapped inside a two-dimensional (2D) harmonic potential, $\nabla_{\perp}^{2} \equiv$ $\partial_{x}^{2}+\partial_{y}^{2}$ is the transverse (2D) Laplacian and $r^{2} \equiv x^{2}+y^{2}$. In fact, the above equation has the form of a "modified" Gross-Pitaevskii equation (GPE), which is the traditional lowest-order mean-field model describing atomic $\mathrm{BECs}^{36,37}$ : the differences of Eq. (11) from the traditional form of the GPE can be traced in the presence of (i) the spatially dependent gain term with

$$
\chi(r)=\alpha \Theta\left(r_{m}-|r|\right),
$$

where $\Theta$ is the step function generating a symmetric spot of radius $r_{m}$ and strength $\alpha$ for the gain, and (ii) the nonlinear saturation loss term, of strength $\sigma$. Estimates of the relevant physical time and space scales, as well as physically relevant parameter values, are given, e.g., in Ref. 24. It is relevant to mention that although our results below are given in the context of Eq. (11), we have ensured that similar phenomenology arises in the model of Refs. 21 23, for suitable parametric choices. In that light, the phenomenology that is reported in this work should be broadly relevant to (2D) polariton BECs independently of model specifics. We also note in passing that Ginzburg-Landau-type models, similar to the one of Eq. (1) -i.e., including a localized gain term (but, in most cases, in the one-dimensional setting and without the external potential) — were recently studied in the context of nonlinear optics 38 and in the physics of magnon condensates 39 .

In what follows, we will consider the stationary solutions of this 2D model, in the form $\psi(r, t)=$ $\psi_{0}(r) \exp (-i \mu t)$ where $\mu$ is the dimensionless chemical potential, and the stationary state $\psi_{0}(r)$ is governed by the elliptic partial differential equation of the form:

$$
\mu \psi_{0}=\left\{-\nabla_{\perp}^{2}+r^{2}+\left|\psi_{0}\right|^{2}+i\left[\left(\chi(r)-\sigma\left|\psi_{0}\right|^{2}\right)\right]\right\} \psi_{0} .
$$

Importantly, an additional population balance constraint, i.e., an overall balancing of gain and loss within the 2D domain, has to be enforced: this condition is $d N / d t=0$, where the norm $N=\int \mathrm{d}^{2} r\left|\psi_{0}\right|^{2}$ represents the number of polaritons. It is straightforward to show that the balance condition can be readily expressed as:

$$
\int \mathrm{d}^{2} r\left(\chi(r)-\sigma\left|\psi_{0}\right|^{2}\right)\left|\psi_{0}\right|^{2}=0 .
$$

It then follows that the above equation self-consistently selects the particular value of the chemical potential once the other parameters (i.e., $\alpha, \sigma$, and $r_{m}$ ) are fixed. We note in passing the significant difference of this trait from 
the Hamiltonian atomic BEC case, where there exist monoparametric families of solutions as a function of $\mu$ (which is a free parameter there rather than one dependent on the remaining gain/loss parameters). Hence, in the computation of the system, it is critical to enforce the condition (4), as this condition will determine in addition to the profile $\psi_{0}$, the associated value of $\mu$.

Once stationary solutions of the differential-algebraic system of Eqs. (3)-(4) are identified, their linear (spectral) stability is considered by means of a Bogolyubov-de Gennes (BdG) analysis ${ }^{36,37}$. Specifically, small perturbations (of order $\mathcal{O}(\delta)$, with $0<\delta \ll 1$ ) are introduced in the form

$$
\psi(x, y, t)=e^{-i \mu t}\left[\psi_{0}(x, y)+\delta p(x, y, t)\right]
$$

with

$$
p(x, y, t) \equiv a(x, y) e^{-i \omega t}+b^{\star}(x, y) e^{i \omega^{\star} t}
$$

Then, the ensuing linearized equations are solved to $\mathcal{O}(\delta)$, leading to the following eigenvalue problem:

$$
\omega\left(\begin{array}{c}
a(x, y) \\
b(x, y)
\end{array}\right)=\left(\begin{array}{cc}
L_{1} & L_{2} \\
-L_{2}^{*} & -L_{1}^{*}
\end{array}\right)\left(\begin{array}{c}
a(x, y) \\
b(x, y)
\end{array}\right)
$$

for the eigenfrequency $\omega$ and associated eigenvector $(a(r), b(r))^{T}$, and $L_{1}$ and $L_{2}$ are the following operators:

$$
\begin{aligned}
& L_{1}=-\mu-\frac{d^{2}}{d x^{2}}-\frac{d^{2}}{d y^{2}}+r^{2}+2(1-i \sigma)\left|\psi_{0}\right|^{2}+i \chi(r), \\
& L_{2}=(1-i \sigma) \psi_{0}^{2} .
\end{aligned}
$$

When the eigenfrequencies are found to possess a positive imaginary part, then, per the ansatz of Eq. (6), an instability is expected to arise. On the other hand, if all the spectrum has $\operatorname{Im}(\omega)<0$, then the corresponding structure is spectrally stable. When a structure is found to be unstable, we conduct direct numerical simulations of Eq. (1) in order to explore the evolution of the instability and the state towards which the dynamics is attracted.

We now proceed to study the existence, stability and nonlinear dynamics of the different configurations of interest, namely the nodeless cloud, the single-charge vortex and the ring dark soliton-like waveform, as well as of some symmetry-breaking structures that result from the evolution dynamics of these states, when unstable.

\section{NUMERICAL RESULTS}

\section{A. Existence and Spectral Stability}

\section{Nodeless Cloud and Central Vortex}

We performed a search of nonlinear excitations for different values of the parameters. In what follows, we chose to keep $\sigma=0.35$ (following the work of Ref. 40) fixed,

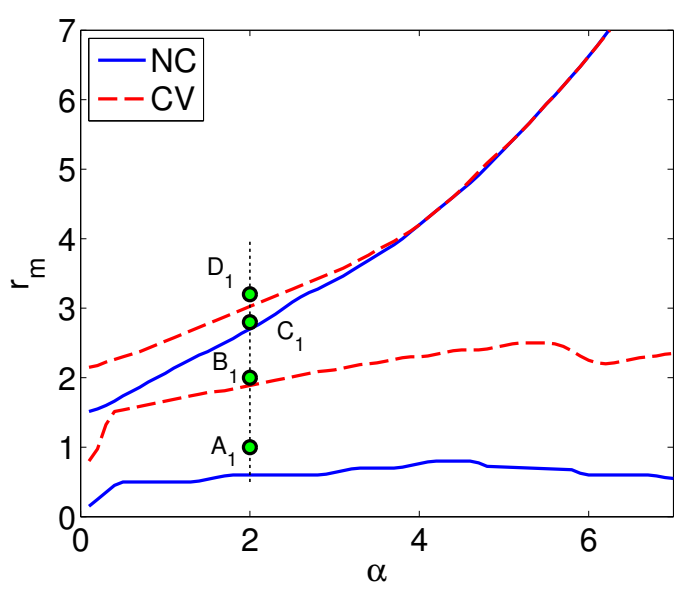

FIG. 1: (Color online) Stability domains of nodeless cloud (NC), and central vortex (CV) soliton-like solutions for $\sigma=0.35$. The stable domains correspond to the regions between the curves. In a wide range of values within the two-dimensional parameter space, these solutions co-exist and may even be concurrently stable. Circles indicate points shown in following figures for $\mathrm{NC}$ and $\mathrm{CV}$ solutions. All quantities shown here, and in the figures that follow, are dimensionless. Curves in this figure (as well those in Figs. 5 and 7 below) were smoothed from data with stepsizes of $\Delta \alpha=0.1$ and $\Delta r_{m}=0.1$, or lower.

and vary both the gain strength, $\alpha$, and the gain spot size radius, $r_{m}$, in order to develop two-parameter bifurcation diagrams characterizing the stability properties of the different states of interest. The relevant solutions were numerically obtained by using a (modified) NewtonRaphson method $\underline{41}$. This is done in order to identify (and perform continuations on) solutions of Eq. (3), together with condition (4). This system forms a partial differential algebraic set of equations (PDAE).

We start by exploring the more fundamental solution profiles, namely the nodeless cloud (NC) and the central vortex cloud $(\mathrm{CV})$.

It would be relevant to recall here, for comparison purposes, the stability properties of these waveforms in the Hamiltonian case of $\alpha=\sigma=0$. There, the NC is the ground state of the system and is neutrally stable for all parameter values 36.37 . Similarly, and although it is an excited state of the system (bearing an "anomalous" or "negative energy" mode), the CV is generically stable, independently of the chemical potential (or effectively the number of atoms) of the system $\underline{42}$.

The results of the scan of the parameter space are represented in Fig. 1, where we show the limits of stability of the $\mathrm{NC}$ and $\mathrm{CV}$. Interestingly, it can be observed that while there are wide parametric regimes where the $\mathrm{NC}$ is stable, there are also large intervals of parameters where this solution is, in fact, unstable, contrary to what is known to be the case in atomic BECs. Furthermore, the stability region for the $\mathrm{NC}$ configuration is bounded both from above and from below, unlike the one-dimensional 

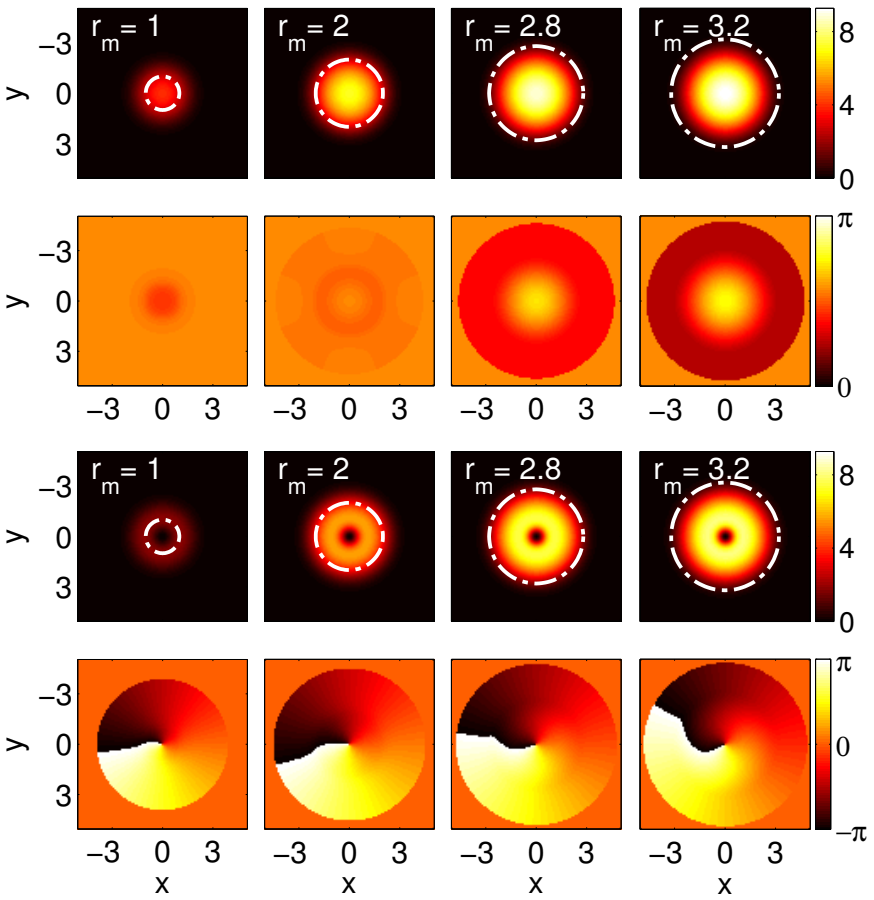

FIG. 2: (Color online) Density and phase profiles of nodeless cloud configurations (NC, top two rows) and central vortex configurations ( $\mathrm{CV}$, bottom two rows) for $\alpha=2.0$ and $\sigma=$ 0.35. The values of $\alpha$ and $r_{m}$ used correspond, left to right, to points $\mathrm{A}_{1}-\mathrm{D}_{1}$ in Fig. 1. Dash-dotted circles, from here on, indicate the boundary of the gain spot.

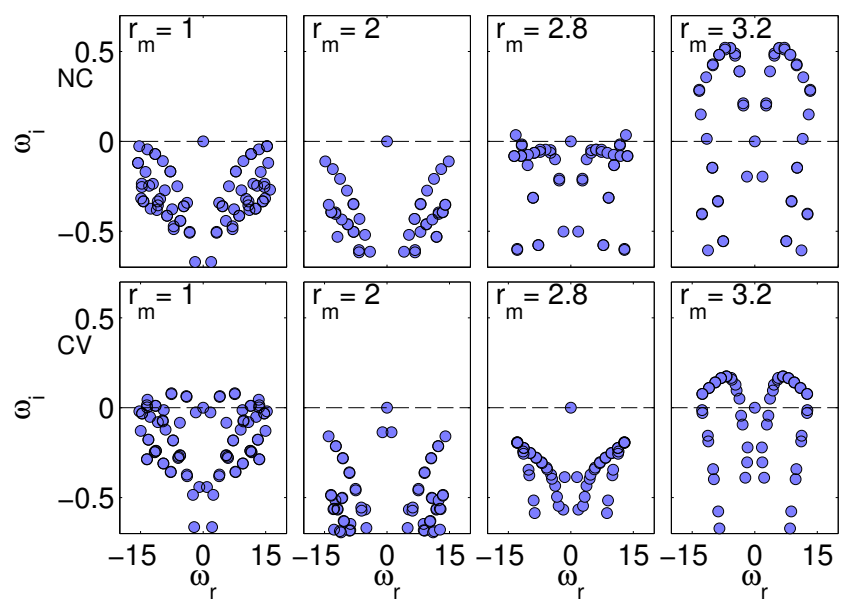

FIG. 3: (Color online) Eigenfrequencies $\left(\omega=\omega_{r}+i \omega_{i}\right)$ associated with the spectral analysis of the NC (top) and CV (bottom) waveforms for the same parameter values as in Fig. 2 Notice how the instability of both the $\mathrm{NC}$ and the CV in these cases emerges from a band of continuous spectrum crossing into the unstable half-plane, as $r_{m}$ is increased. This predisposes us towards a "dramatic" instability evolution that will significantly modify the background state and will result, as we will see below, in the formation of vortex lattice configurations.
(1D) scenario of Ref. 40 where the stability region is only bounded above. Since there is loss everywhere, as the spot size goes to zero there is only enough gain to sustain a progressively smaller condensate, until it disappears in the limit of $r_{m}=0$. On the other hand, also the CV features wide intervals of stability, but also ones of instability. It can, in fact, be seen that the feature identified as "stability inversion" in Ref. 40 for the case of $1 \mathrm{D}$ polariton BECs is still present here. Namely, there are regimes where the $\mathrm{NC}$ is stable but the $\mathrm{CV}$ is not, but also - in reverse - there are regimes where the $\mathrm{CV}$ is stable, but the NC is not.

In Fig. 2 we show the density and phase profiles of the $\mathrm{NC}$ (top two rows) and CV (bottom two rows) solutions for varying $r_{m}$. It is clear that as the radius of the drive increases, so does the size of the condensate. This is in contrast to what is the case with atomic BECs, where the size of the $\mathrm{NC}$ is controlled solely by the (parabolic) trap: here, the gain (and its interplay with the nonlinear loss/saturation) plays a critical role in the size of the waveform. For the CV solutions of the bottom two rows, notice the characteristic $2 \pi$ phase circulation.

We now turn to an examination of the stability of the different configurations. The spectral planes $\left(\omega_{r}, \omega_{i}\right)$, where the subscripts $r$ and $i$ denote, respectively the real and imaginary parts of the eigenfrequency, for the $\mathrm{NC}$ and the $\mathrm{CV}$ configurations are illustrated in Fig. 3 . There, it is evident that except for a weak instability arising through Hopf bifurcations for small values of $r_{m}$, for most intermediate values of $r_{m}$, both the $\mathrm{NC}$ and the CV configuration are stable. The predominant instability that arises for both configurations is the one for higher values of the gain radius $r_{m}$ in this continuation. In that case, the instability arises in a less customary (for such structures, at least in their Hamiltonian form) way: entire segments of the continuous spectrum cross over the axis of $\omega_{i}=0$ (see right panels of Fig. 3) and lead to bands of unstable eigenfrequencies. It is, thus, in a sense, perhaps expected that the entire "background state" of the system will be highly unstable towards a fundamentally different pattern, an expectation that indeed we will see to be confirmed by the direct numerical simulations featuring the instability evolution of these states.

\section{Gray Ring Solitons and Triangular States}

In addition to the more fundamental solutions explored above, we have identified a host of previously undisclosed, to the best of our knowledge, solutions of the 2D polariton BEC system ${ }^{34}$. Arguably, the most remarkable among them is the gray ring (GR) soliton, some typical density profiles of which are shown in Fig. 4. The depicted profiles correspond to the points shown as (green) dots in the 2D existence/stability $\left(r_{m}, \alpha\right)$-plane (i.e., width and amplitude of the parametric gain) shown in Fig. 5. The plane itself reveals some of the interesting potential of such solutions, including the possibility of 


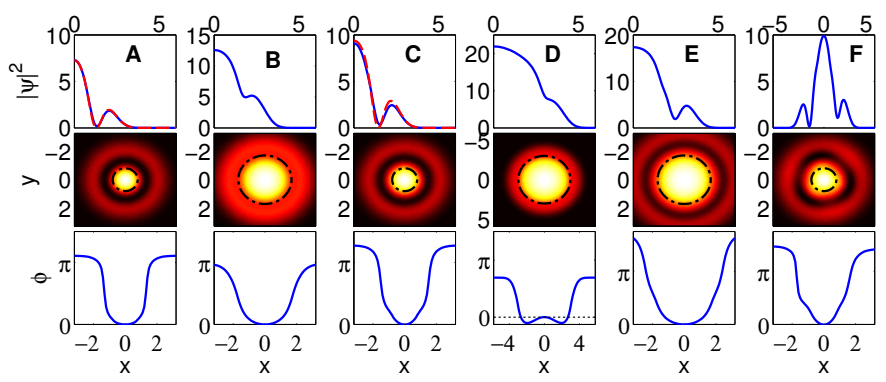

FIG. 4: (Color online) Profiles (upper two rows) and phase (bottom row) of gray ring (GR) solitons, and triangular solutions (TS) for the system parameters marked by (green) dots in the 2D plane of Fig. 5 . $\sigma=0.35$. Dashed (red) lines represent the Hamiltonian dark ring for the same chemical potential (panels A and C). Panel F represents a TS solution. Notice its asymmetry both in density and phase.

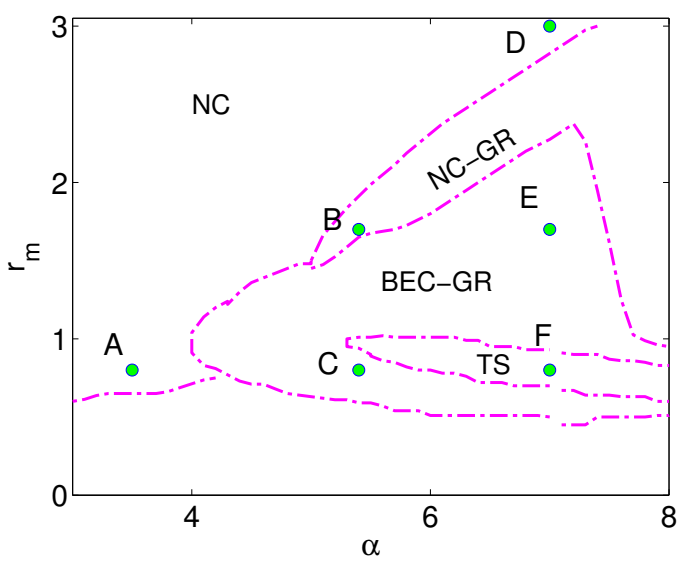

FIG. 5: (Color online) Existence domains of gray ring (GR) solitons and triangular solutions (TS) for $\sigma=0.35$. Solutions are stable except that the GR is unstable where the TS exists. For the distinction between GR solitons arising from the Hamiltonian BEC limit (BEC-GR) and those emerging from the NC (NC-GR), see details in the text.

symmetry-breaking bifurcations giving rise to a new triangular form of solutions (denoted as TS). An additional remarkable feature is that while such ring dark solitons were proposed in both atomic condensates $29-31$ and in cGL gain/loss systems (as radial Nozaki-Bekki holes) in both contexts they were found to be unstable and thus break up into more prototypical coherent structures, including vortices and spiral waves, respectively. We now turn to a more detailed examination of their properties including the existence and stability domains, also highlighting similarities and importantly differences from the above atomic case, including the generically gray nature of such excitations in our polariton setting.

For relatively low values of the gain spot size $\left(r_{m}<\right.$ 1.3) the GR soliton is always unstable for $\alpha<4.0$, but can be stable above that gain value. It coexists with the
NC over most of this spot size range.

Above that spot size value, the GR soliton solution can only be identified within the parametric range indicated by the curves in Fig. 5 .

Interestingly, what is illustrated by the parametric plane is a progressive convergence of the GR and $\mathrm{NC}$ solutions, as represented by the label NC-GR in Fig. 5 . This gradual "merging", at the solution profile level, is signaled by the progressive increase of the phase variation of the NC until it reaches a value around $\pi$. Recall that from the results presented for the NC branch (Fig. 2), the phase varies by less than $\pi / 2$.

The admittedly somewhat arbitrary distinction between the NC branch and the GR one (and their hybrid NC-GR form) herein was based on whether there is a dip in the density profile (NC-GR) or not (NC). In particular, we observe that as the amplitude $\alpha$ of the gain grows, the NC becomes progressively more modulated (as already seen and explained in Ref. 24).

To be more precise, we point out that the distinction between accordingly termed BEC-GRs and the previously discussed NC-GRs is obtained through the nonmonotonic dependence of their dip versus the gain radius $r_{m}$. More specifically, for GRs, the depth of their dip (measured as the difference of the density at the center minus the density at the dip) is found to increase with increasing $r_{m}$, while NC-GRs are instead characterized by a decreasing dip (and NCs by a non-existent one). In reality, these solutions seem to seamlessly merge as the critical points identified are traversed, however, the above distinctions were given in order to better appreciate the "origin" of the different solutions.

In fact, there is an additional connection of such GR soliton solutions with their BEC analogues discussed earlier in Refs. 29 31. In particular, as we approach the limit of weak and narrow drive ( $\alpha \rightarrow 0$ and $r_{m}$ small), the solution increasingly resembles the BEC ring dark soliton (RDS) of the above works. This is illustrated in panels $\mathrm{A}$ and $\mathrm{C}$ of Fig. 4 where, in addition to the polaritonic GR soliton profile, the corresponding Hamiltonian case is also shown for the same chemical potential. As can be seen, the dip in both cases occurs at the same position, that is distinct from the gain spot radius. This (and the overall quality of the density comparison) is a strong indication of the common origin of this solution with its Hamiltonian sibling. Hence the label BEC-GR in Fig. 5 and in the discussion above. Recalling that the RDS in the Hamiltonian case is always unstable, we identify herein a critical role of the gain along with the saturating loss terms in inducing a limited region where this GR soliton can be stabilized.

Thus, the origin of the NC and GR solutions is indeed distinct, as in the BEC-GR case, the solutions physically emerge as a result of the interplay of the linear potential and its modes with the effective nonlinearity of the system. On the other hand, in the NC-GR case, the gain (and loss) structure of the system plays a critical role in inducing a flux pattern that, in turn, creates the observed 


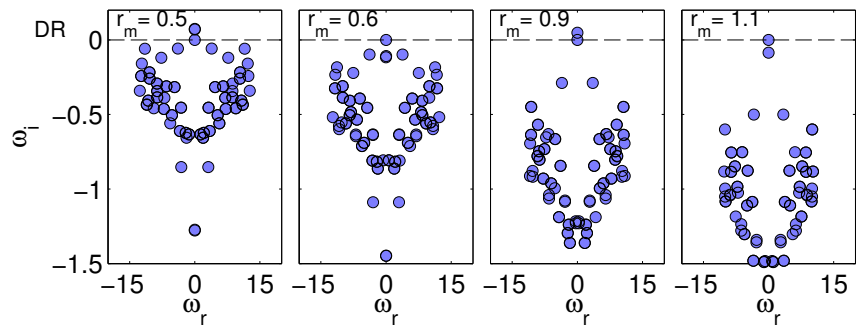

FIG. 6: (Color online) Spectra pertaining to the GR soliton solution for $\alpha=6.0$. Notice that for $r_{m}=0.9$ the unstable GR solution coexists with a stable TS. This is another example of the co-existence of solutions discussed above for the same parameter values. Notice how these spectra differ from the earlier shown ones in Fig. 3 in that the instability arises from a point spectrum mode crossing through the origin and giving rise to the formation of a symmetry broken state (the TS).

dip in the density profile.

In the light of our distinction, the density profiles observed in Ref. 24, that exhibit a gradual deepening of the shoulder (e.g. as $\alpha$, the strength of the gain, is increased) are still NCs and not GRs. Thus, the former observations can be considered "precursors" of our GR states (but not examples of ones such).

An additional comment should be made here about the gray nature of these rings. Contrary to what may be expected from their BEC siblings featuring a phase shift of $\pi$ when stationary (and associated with a finite velocity when they are "gray"), in the polaritonic case, the rings are generically found to be gray. This is, in fact, reminiscent of what was recently found also in the context of complex $\mathcal{P} \mathcal{T}$-symmetric potentials, e.g., in the work of Refs. 43, 44. In both cases, the origin of the phenomenon is the same: in particular, the complex nature (of the potential in the $\mathcal{P} \mathcal{T}$-symmetric case and of the gain/loss structure in the cGL setting herein) of the terms in the equation produce a genuinely complex solution with a non-trivial phase structure and an associated "particle flux" along the stationary spatial profile. These features are absent in the BEC case, where the stationary RDS solution is genuinely real.

We note in passing that we found another radially symmetric soliton solution resembling the GR soliton. This solution has its dip closer to the gain spot size, but it was never found to be stable. It is therefore related to this length scale, contrary to what is the case for the GR solutions focused upon here. Due to the generic instability of the waveform apparently slaved to the gain, we do not explore it further here.

The spectral properties of the GR soliton state have been found to be significantly different than those of the previous two fundamental states (NC and $\mathrm{CV}$ ). In particular, as can be seen in Fig. [6] the instability of this state as $r_{m}$ is increased stems from the fact that eigen- frequencies cross the origin of the spectral plane. This predisposes us towards a fundamentally different instability, possibly arising through a symmetry breaking pitchfork bifurcation. We will see also how this expectation is manifested in the direct numerical simulations of the following section. This symmetry breaking bifurcation is substantiated in the parametric plane of Fig. 5 where a region has been denoted under TS (triangular solutions). As the curve outlining this region is crossed, the BEC-GR soliton solutions undergo the above mentioned pitchfork bifurcation and spontaneously give rise to such TS. The TS states are generically found to be stable, a feature that will render them natural attractors for the BECGR soliton unstable dynamics in the parametric range of TS existence, as will be corroborated in the dynamical evolution section below.

\section{Quadrupoles}

In the same spirit as the triangular solutions identified above, we have also been able to find solutions with quadrupolar symmetry. These solutions are especially relevant below the region of stability of the $\mathrm{NC}$, as well as that of the (BEC-)GR. Such solutions are characterized by four dips of the density, at which locations the phase portrait shows a winding of $2 \pi$ (Fig. 8). This type of solution is also reminiscent of a corresponding quadrupole (vortex) solution in the realm of atomic BECs 42 . In the latter setting, the solutions are critically induced by the parabolic trap, created from the linear limit thereof (as a complex combination of two of the second excited states of the 2D quantum-harmonic oscillator).

The regions of stability of the quadrupoles in the two parameter plane of $\left(\alpha, r_{m}\right)$ are shown in Fig. 7 . Different classes of instability can be found, leading to exponential, oscillatory or combined decay. Nevertheless, islands of stability are also identified within which as we will see below the quadrupolar state can offer a dynamical attractor starting, e.g., from GR soliton initial data, but also for both the $\mathrm{NC}$ and the $\mathrm{CV}$.

As the gain strength grows, we find that the quadrupole exact solution profiles tend to a more elongated profile along a symmetry axis, reaching a form where the four dips nearly coalesce in two. Nevertheless, the phase (and the vorticity, not shown) clearly show that the four vortices of alternating positive and negative charge are still separate.

\section{B. Dynamical evolution}

As a way of confirming the stability results found above, and also of exploring the pattern forming outcomes of the dynamical evolution of the identified instabilities, we numerically integrated the full equation of motion, namely Eq. (11). Our initial conditions consisted of profiles in the form of the above obtained exact (up 


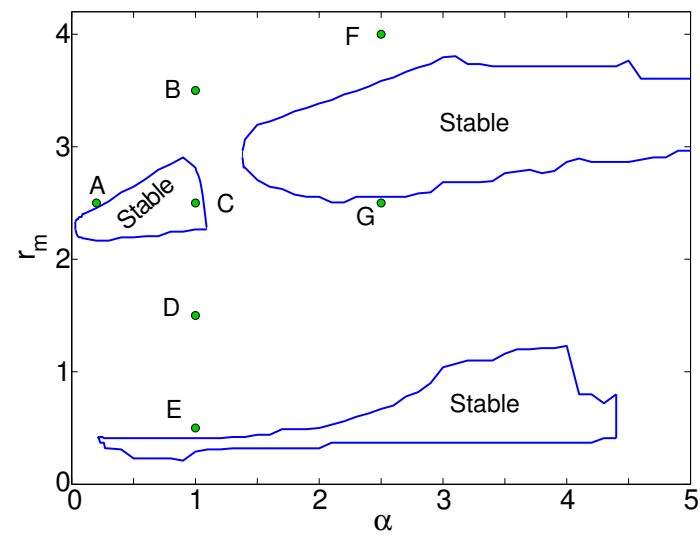

FIG. 7: (Color online) Stability domains of quadrupole solutions (QS), for $\sigma=0.35$. The different letters denote case example profiles illustrated below in Fig. 8
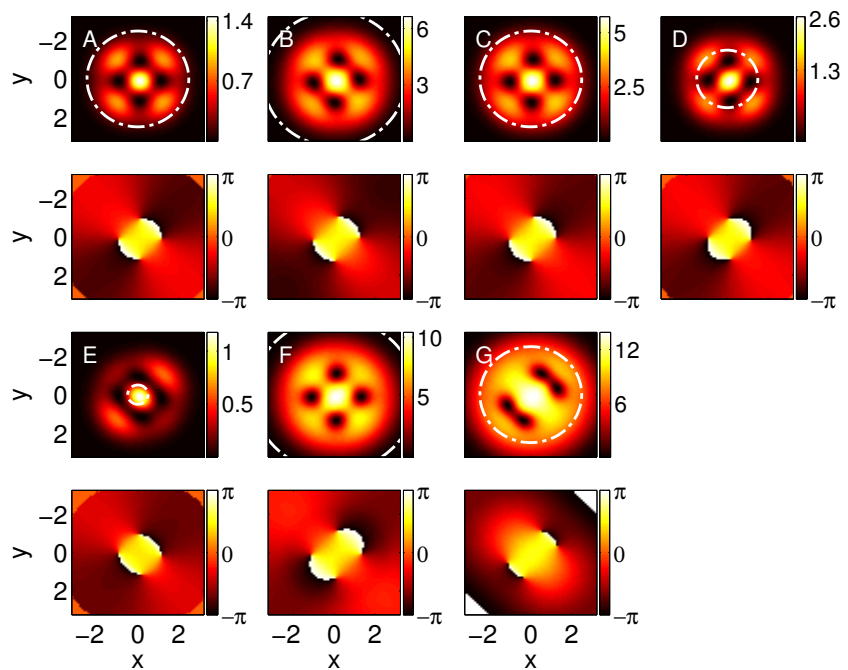

FIG. 8: (Color online) Density and phase profiles of quadrupole solutions (QS) for the values of $\alpha$ and $r_{m}$ corresponding to points $\mathrm{A}-\mathrm{G}$ in Fig. 7

to a prescribed numerical tolerance) solutions, suitably perturbed to accelerate the decay, if unstable, or confirm that it returns to the attracting solution, if stable. The perturbation added for unstable waveforms was in the form of the profile of the eigenvector with the most unstable eigenfrequency. For the solutions expected to be stable, random noise was used. The latter results are not shown (they were only used to confirm the spectral stability results), but it was found that the solutions remained unaltered after propagation for time up to $t=1000$.

Examples of the unstable scenarios are presented below $\underline{45}$. The results presented correspond again to points of the set $A_{1}-D_{1}$ (see Fig. 1) that are unstable (for each of $\mathrm{NC}$ and/or $\mathrm{CV}$ ); other values lead to a qualitatively similar behavior. Figure 9 illustrates the case
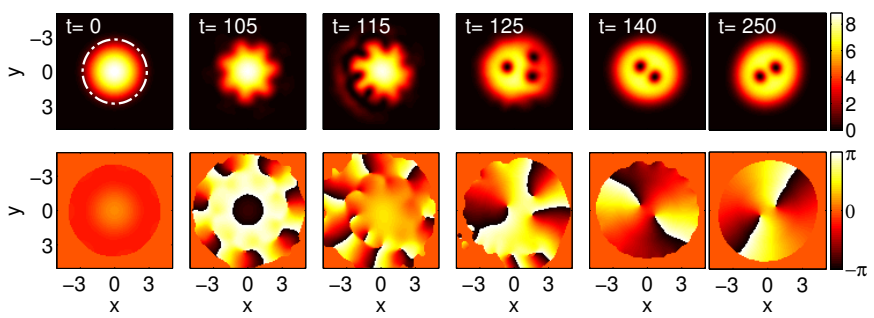

FIG. 9: (Color online) Dynamical evolution of a NC state for parameter values $\alpha=2.0$ and $r_{m}=2.8$, which is just outside of the stability region (see point $\mathrm{C}_{1}$ in Fig. 1).
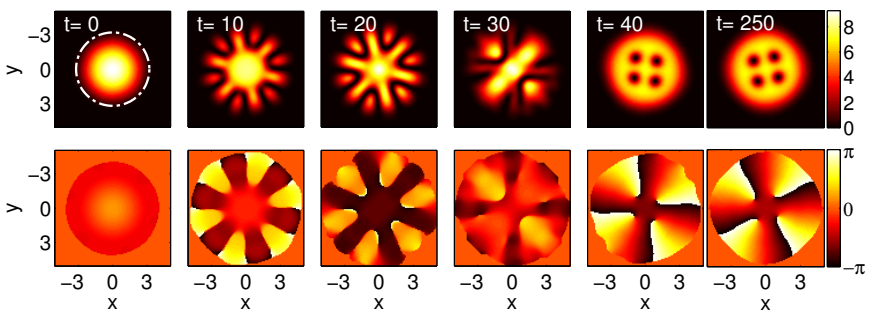

FIG. 10: (Color online) Dynamical evolution of a NC state for parameter values $\alpha=2.0$ and $r_{m}=3.2$ (see point $\mathrm{D}_{1}$ in Fig. 11). The resulting configuration of 4 vortices is rotating.

where the NC has just become unstable (for $r_{m}=2.8$, while the stability boundary for this value of $\alpha=2$ is $\left.r_{m}=2.7\right)$. In this case, we can observe that the NC becomes unstable to an azimuthal modulation with eightfold symmetry and eventually, upon the nonlinear evolution of the instability, decays to a pair of rotating vortices. A further increase of $r_{m}=3.2$ results in a similar evolution (cf. Fig. 10), but the end result is a rotating lattice of four vortices. Here we see a different initial symmetry in the dominant unstable mode (which appears to create a hexagonal modulation; see, e.g., the snapshots at $t=10$ and $t=20$ ), but also a nonlinear intermediate step in the evolution (see, e.g., snapshot at $t=30$ ). It is naturally expected that, as $r_{m}$ increases and the polariton condensate accordingly grows, more vortices can be "accommodated" therein, i.e., brought in the domain from the outside, and hence larger lattices created; this is in line also with the observations of Ref. 24. The evolution typically starts with a modulation around the edge of the cloud that starts rotating. Comparison with the profile of the most unstable eigenvector suggests that the latter is indeed responsible for this (increasing) modulation. It eventually leads to one or more vortices spiralling (from the periphery) to the central region of the cloud until a stable and symmetric arrangement of vortices is achieved. The number of vortices (in the parameter range studied) can vary from one up to 21 , either in a ring shape, with one more at the center, or as a lattice when their number grows.

The evolution of the CV solution features a similar be- 


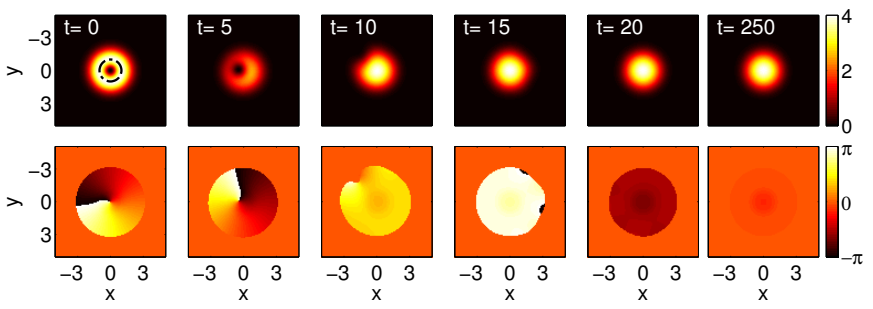

FIG. 11: (Color online) Dynamical evolution of a CV state for parameter values $\alpha=2.0$ and $r_{m}=1.0$, which is below its stability region (see point $A_{1}$ in Fig. 1).
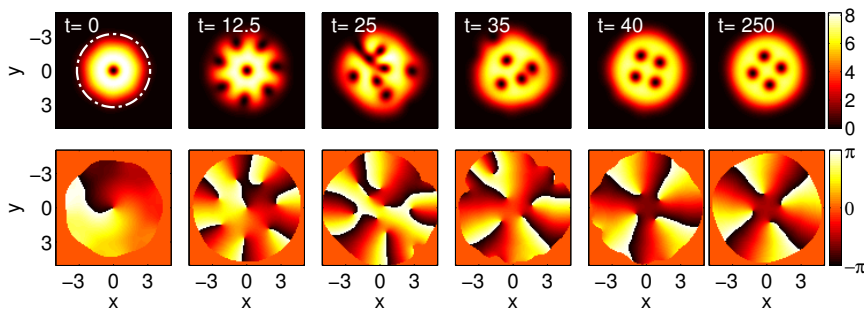

FIG. 12: (Color online) Dynamical evolution of a CV state for parameter values $\alpha=2.0$ and $r_{m}=3.2$, which is just outside (above) its stability region (see point $\mathrm{D}_{1}$ in Fig. 1). The resulting configuration appears to ultimately lead to a rotating square (cf. Fig. 10 for the same parameter values).

havior to the $\mathrm{NC}$ for parametric values beyond the upper stability boundary of this solution. In the region below the lower stability boundary, where the $\mathrm{NC}$ is generically stable, the CV typically decays to the $\mathrm{NC}$ (see, e.g., Fig. 11). Above the higher stability boundary of the CV solution, the vortex decays to a lattice of vortices as shown in Fig. 12, It is interesting to note that the original evolution of the instability results in more vortices within the cloud than the resulting asymptotic state, so there is a "distilling" process taking place, which finally results in the rotating square configuration observed at longer times (cf. for the same parameters, the asymptotically favored configuration of Fig. 1010.

We now turn to the dynamical results for the evolution

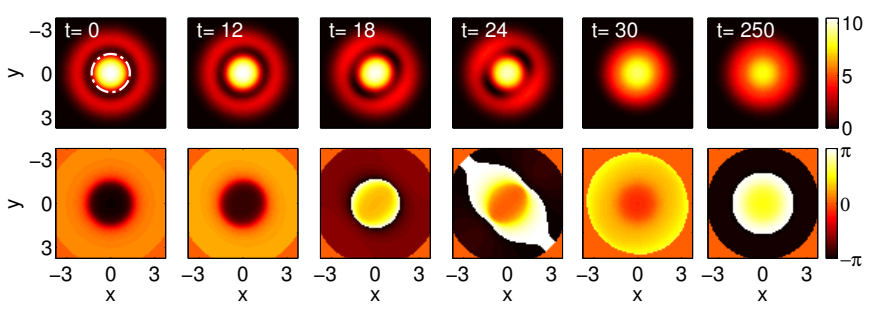

FIG. 13: (Color online) Dynamical evolution of a GR soliton state for parameter values $\alpha=4.2$ and $r_{m}=1.3$, which is above its stability region. The final excitation is a NC state.
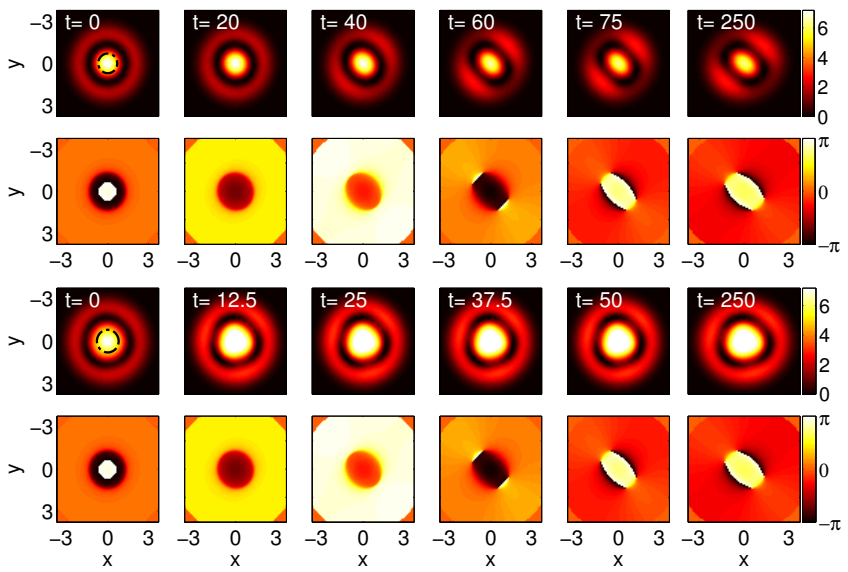

FIG. 14: (Color online) The top two rows show the dynamical evolution of a GR soliton state for parameter values $\alpha=4.2$ and $r_{m}=0.7$, which is below its stability region. The final excitation features axial symmetry and is a QS. The bottom two rows show the dynamical evolution of a GR soliton state for parameter values $\alpha=6.9$ and $r_{m}=0.8$, which is also outside its stability region. Here, however, the final excitation features triangular symmetry (TS).

of the GR soliton solutions. Above its stability region, the GR soliton typically decays towards the NC, which for this parametric region is well within its own range of stability in the $\left(\alpha, r_{m}\right)$ plane; this is shown, e.g., in Fig. 13, for $\alpha=4.2$ and $r_{m}=1.3$. In contrast, below the lower stability threshold of the GR, the latter may decay to different solutions depending on the exact parameters (and perturbations) used. An example of a relevant possibility is shown in the top two rows of Fig. 14 for $\alpha=4.2$ and $r_{m}=0.7$. This case reveals the possibility that the symmetry breaking instability of the GR soliton (cf. the discussion of the previous section) may result into a quadrupolar configuration of the type explored in the previous section. On the other hand, the bottom two rows of Fig. 14 illustrate a different scenario for the parameter set $\alpha=6.9$ and $r_{m}=0.8$, which can be identified as being within the region of the symmetry breaking instability towards triangular solutions. In particular, the symmetry breaking spontaneously manifests itself dynamically resulting towards a configuration with triangular symmetry, as may be expected based on our existence/stability earlier findings. Solutions with this symmetry were previously identified in other contexts, e.g., Ref. 46. Nevertheless, these results, and the absence of vortex lattice formation in this case, are in accordance with the results of the stability analysis of the GR soliton presented above and its fundamentally different instability mechanism in comparison to the NC or CV configurations.

The evolution of the QS solutions shows a range of behaviors, from a decay towards a NC (for points D and G in Fig. 77) to rotating lattices of vortices (A, B, C, and F). Among these, we highlight, in particular, the scenario A 

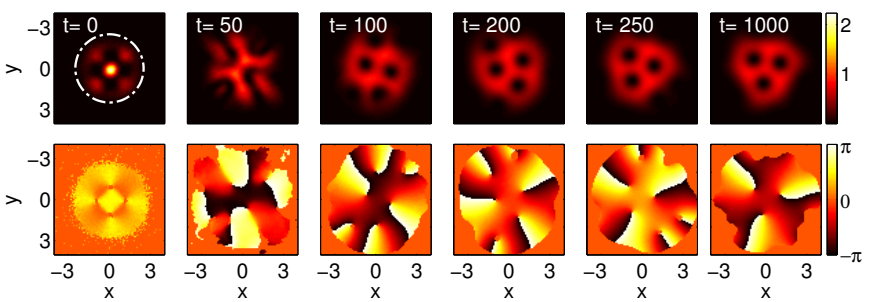

FIG. 15: (Color online) Dynamical evolution of a QS for parameter values $\alpha=0.2$ and $r_{m}=2.5$ (see point labeled $\mathrm{A}$ in Fig. 9). The final state is a rotating lattice of 3 vortices.
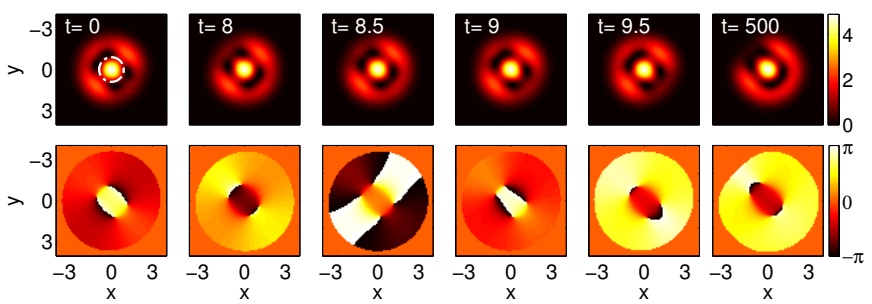

FIG. 16: (Color online) Dynamical evolution of a QS for parameter values $\alpha=2.5$ and $r_{m}=0.9$ (not in Fig. 7). It corresponds to an oscillatory instability.

( $\left.\alpha=0.2, r_{m}=2.5\right)$, shown in Fig. 15, where the final rotating cloud is highly distorted, as if more vortices were trying to join the 3 already in the central region of the cloud. It can also display an oscillatory instability, as in Fig. 16] (see relevant movie provided in the Supplementary Material, where this instability is more transparently demonstrated), where the two dips at the extrema of the axial central lobe perform an oscillation, each pair with opposite phase than the other. Yet another situation is exemplified in Fig. 17, where a lattice of vortices results, but unlike all other so far shown herein the inner vortices rotate at a different rate than the outer ones. Finally, the case F (see Fig. 18) results in an excitation where a lattice with 7 vortices (one at the center and an hexagon of vortices around it) rotates very slowly.

We should remark here that the vortex lattices (with different numbers of vortices) observed herein through the unstable dynamical evolution of our states correspond to the same states as reported earlier in Ref. 24 and further elaborated, e.g., in Ref. 26. Nevertheless, our aim here is to show that such states arise from the unstable dynamics of not only fundamental states (such as the $\mathrm{NC}$ and $\mathrm{CV}$ ) but also from more complex states such as the quadrupole ones. In addition, our scope is to also show that in regimes of drive where such lattices may exist, it may also be possible to dynamically result in other states including e.g. the ones with triangular symmetry (as, e.g., in Fig. 14).
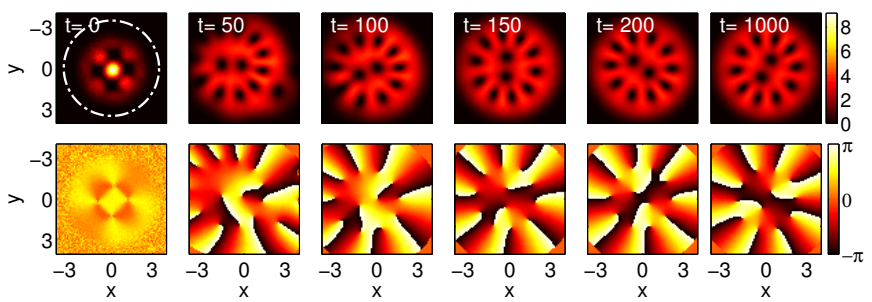

FIG. 17: (Color online) Dynamical evolution of a QS for parameter values $\alpha=1.0$ and $r_{m}=3.5$ (see point labeled B in Fig. (7). The final state is a rotating lattice of 10 vortices in a necklace plus 2 at the center. The two sets rotate at different rates. (Initial frame has its colormap scaled by a factor of $1 / 5)$.
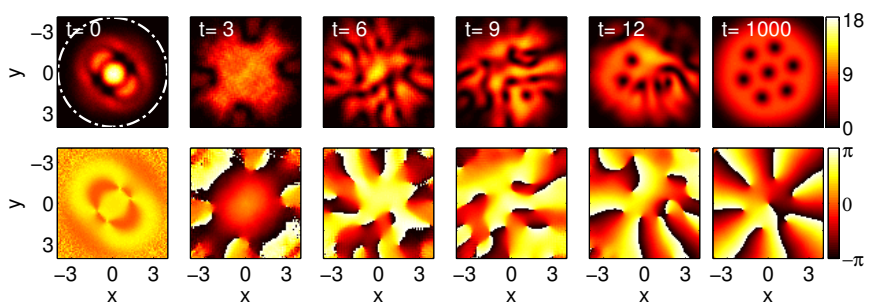

FIG. 18: (Color online) Dynamical evolution of a QS for parameter values $\alpha=2.5$ and $r_{m}=4.0$ (see point labeled $\mathrm{F}$ in Fig. 7). The final state is a (slowly) rotating lattice of 7 vortices. (Initial frame has its colormap scaled by a factor of $1 / 10)$.

\section{CONCLUSIONS}

In the present work, motivated by the intensely studied theme of polariton condensates, we offered a detailed view of the existence and stability, as well as the nonlinear dynamical properties of some prototypical states appearing in such systems. These states included fundamental earlier revealed waveforms such as the nodeless cloud (NC) and the cloud with a central vortex (CV). For these, we presented a systematic two-parametric analysis of their stability properties and how these are reflected in the corresponding nonlinear dynamics.

The fundamental (especially in the atomic BEC case) nodeless state was found to be stable only in a limited range of parameters. The most simple excited state, the central vortex state (which is again generically robust in atomic BECs), was found to, in fact, potentially exist as a stable object for parameters where the $\mathrm{NC}$ is no longer stable. Vice versa, the nodeless cloud was also stable in regimes where the vortex was not, presenting a $2 \mathrm{D}$ generalization of the stability inversions reported earlier in $1 \mathrm{D}$ counterparts of the mode ${ }^{40}$. Outside their stability ranges, they were both found to decay towards a series of rotating vortex lattices, in line with earlier numerical observations 24 . However, here the precise (Hopf) nature of the instability was elucidated and the unusual 
associated morphology featuring the destabilization of an entire band of continuous spectrum eigenfrequencies was revealed.

As regards vortices, it should also be noted that we attempted to identify doubly charged vortex solutions, however, we were unable to obtain spectrally stable such structures in the realm of the present model, i.e., such states were identified yet were always found to be dynamically unstable.

Such fundamental nonlinear wave states are expected to exist in this system, at a qualitative level, due to the interplay of dispersion and effective nonlinearity (due to the polariton-polariton interaction), as well as that of gain (due to generation of the polaritons from the exciton reservoir) and loss (due to the finite polariton lifetime). However, in our view, it is much harder to qualitatively explain the stability features observed, although some relevant speculation can be offered. For instance, it appears that the vortices can only be stabilized when $r_{m}$ (the radius of the gain spot) is sufficiently large, i.e., it should be able to encompass a vortex core and anyway should be larger than the healing length [of $\mathrm{O}(1)$ in our setup]. On the other hand, for the NC to be robust, we need sufficiently high gain (so as to balance the dissipation-induced loss), as well as a sufficiently large radius of gain drive $r_{m}$ so as to favor the original radial NC structure.

In addition to these simpler structures, we also explored more elaborate ones, especially in the form of a gray ring (GR) soliton structure, which was connected both to the NC but also to the ring dark soliton state of atomic BECs. The inclusion of gain and loss in our complex Ginzburg-Landau-like equation was found to play a critical role in the potential stabilization of such ring states. Their generic gray structure was also justified by the flux induced by the gain/loss. Aside from identifying the stability islands of such GR soliton states, their potential dynamical instabilities and associated bifurcations were also revealed. These were shown to lead to symmetry-breaking events generating (and asymptoting to) solutions of potentially triangular or quadrupolar structure. These states, in turn, were also identified as exact stationary solutions and their own two-parametric stability properties were explored.

A different set of gray ring structures was found but never stable, from what we could determine. This solution is mainly characterized by a central peak lower than the outer ring. It exists in regions in parameter space where other solutions exist stably. Thus for certain re- gions of parameters it is possible to find a NC, a GR and this other, always unstable gray ring soliton.

All the results reported were for the parameter $\sigma=$ 0.35, as stated before. Both from our previous results in the 1D setting, and from intuition and case examples considered, we expect that extending the continuation to other (nearby) values of $\sigma$ yield qualitatively similar results.

There are many directions towards which this exploration could further proceed. On the one hand, in the setting with the parabolic trap, it is interesting to explore the detailed stability of vortex clusters and progressively growing configurations towards vortex lattices. Understanding these clusters is still a very active area of research in atomic $\mathrm{BECs}^{47}$; extending relevant (vortex) particle approaches (or distributional ones ${ }^{48}$ ) in order to understand the properties and internal modes (in analogy to the Tkachenko modes of atomic $\mathrm{BECs}^{49}$ ) of the system of a few or of many vortices, would be of particular interest in its own right. Yet another interesting direction, given the significant progress in imposing potentials of different kinds including periodic ones and identifying states critically supported by them (such as the gap solitons of Ref. 18), would be to explore the interplay of such clusters and lattices with external potentials and their structural phase transitions between different energetically preferred states as, e.g., the lattice parameters of an external periodic potential are varied ${ }^{50}$. These and other related topics are presently under consideration and will be reported in future publications.

\section{Acknowledgments}

P.G.K. gratefully acknowledges support from the National Science Foundation, under grant CMMI-1000337, from the AFOSR under grant FA9550-12-1-0332, from the Binational Science Foundation under grant 2010239 and finally the warm hospitality of the IMA of the University of Minnesota and of KIP and the University of Heidelberg during the final stages of this work. A.S.R. also acknowledges the hospitality of KIP and of the University of Heidelberg. The work of D.J.F. was partially supported by the Special Account for Research Grants of the University of Athens. J.C. and F.P. acknowledge financial support from the MICINN project FIS200804848 .
1 J. Kasprzak, M. Richard, S. Kundermann, A. Baas, P. Jeambrun, J. Keeling, F.M. Marchetti, M.H. Szymańska, R. André, J.L. Staehli, V. Savona, P.B. Littlewood, B. Deveaud, and L.S. Dang, Nature 443, 409 (2006).

2 R. Balili, V. Hartwell, D. Snoke, L. Pfeiffer, and K. West, Science 316, 1007 (2007).
${ }^{3}$ W. Lai, N.Y. Kim, S. Utsunomiya, G. Roumpos, H. Deng, M.D. Fraser, T. Byrnes, P. Recher, N. Kumada, T. Fujisawa, and Y. Yamamoto, Nature 450, 529 (2007).

4 H. Deng, G.S. Solomon, R. Hey, K.H. Ploog, and Y. Yamamoto, Phys. Rev. Lett. 99, 126403 (2007).

5 B. Deveaud (Ed.), The Physics of Semiconductor Micro- 
cavities (Wiley-VCH, Weinheim, 2007).

6 Exciton Polaritons in Microcavities, New Frontiers, Springer Series in Solid-State Sciences Vol. 172, edited by D. Sanvitto and V. Timofeev (Springer, New York, 2012).

7 H. Deng, H. Haug, and Y. Yamamoto, Rev. Mod. Phys. 82, 1489 (2010).

8 J. Keeling and N.G. Berloff, Contemporary Phys. 52, 131 (2011).

9 I. Carusotto and C. Ciuti, Rev. Mod. Phys. 85, 299 (2013).

10 A. Amo, J. Lefrère, S. Pigeon, C. Abrados, C. Ciuti, I. Carusotto, R. Houdré, E. Giacobino, and A. Bramati, Nature Phys. 5, 805 (2009).

11 K.G. Lagoudakis, M. Wouters, M. Richard, A. Baas, I. Carusotto, R. André, L.S. Dang, and B. Deveaud-Plédran, Nature Phys. 4, 706 (2008); see also for half-quantum vortices the work of K.G. Lagoudakis, T. Ostatnický, A.V. Kavokin, Y.G. Rubo, R. André, B. Deveaud-Plédran, Science 326, 974 (2009).

12 M.D. Fraser, G. Roumpos, and Y. Yamamoto, New J. Phys. 11, 113048 (2009).

13 G. Roumpos, M.D. Fraser, A. Loffler, S. Höffling, A. Forchel, Y. Yamamoto, Nature Phys. 7, 129 (2011); G. Nardin, G. Grosso, Y. Léger, B. Pietka, F. Morier-Genoud and B. Deveaud-Plédran, Nature Phys. 7, 635 (2011); G. Tosi, F.M. Marchetti, D. Sanvitto, C. Antón, M.H. Szymańska, A. Berceanu, C. Tejedor, L. Marrucci, A. Lemaître, J. Bloch, and L. Viña, Phys. Rev. Lett. 107, 036401 (2011).

14 D. Sanvitto, F.M. Marchetti, M.H. Szymańska, G. Tosi, M. Baudisch, F.P. Laussy, D.N. Krizhanovskii, M.S. Skolnick, L. Marrucci, A. Lemaître, J. Bloch, C. Tejedor, and L. Viña, Nature Phys. 6, 527 (2010).

15 A. Amo, D. Sanvitto, F.P. Laussy, D. Ballarini, E. del Valle, M.D. Martin, A. Lemaître, J. Bloch, D.N. Krizhanovskii, M.S. Skolnick, C. Tejedor, and L Viña, Nature 457, 291 (2009).

16 M. Sich, D.N. Krizhanovskii, M.S. Skolnick, A.V. Gorbach, R. Hartley, D.V. Skryabin, E.A. Cerda-Méndez, K. Biermann, R. Hey, and P.V. Santos, Nature Photonics 6, 50 (2012).

17 A.V. Yulin, O.A. Egorov, F. Lederer, and D.V. Skryabin, Phys. Rev. A 78, 061801 (2008); G. Grosso,G. Nardin, F. Morier-Genoud,Y. Léger, and B. Deveaud-Plédran, Phys. Rev. Lett. 107, 245301 (2011).

18 D. Tanese, H. Flayac, D. Solnyshkov, A. Amo, A. Lemaître, E. Galopin, R. Braive, P. Senellart, I. Sagnes, G. Malpuech, and J. Bloch, Nat. Commun. 4, 1749 (2013); see also for a theoretical analysis of gap solitons in polariton condensates the recent work of E.A. Ostrovskaya, J. Abdullaev, M.D. Fraser, A.S. Desyatnikov, and Yu.S. Kivshar, Phys. Rev. Lett. 110, 170407 (2013).

19 A. Amo, T.C.H. Liew, C. Adrados, R. Houdré, E. Giacobino, A.V. Kavokin, and A. Bramati, Nature Photonics 4, 361 (2010).

${ }^{20}$ S.I. Tsintzos, N.T. Pelekanos, G. Konstantinidis, Z. Hatzopoulos, and P.G. Savvidis, Nature 453, 372 (2008).

21 M. Wouters and I. Carusotto, Phys. Rev. Lett. 99, 140402 (2007).

22 M. Wouters, I. Carusotto, and C. Ciuti, Phys. Rev. B 77, 115340 (2008).

${ }^{23}$ C. Ciuti and I. Carusotto, Phys. Stat. Sol. (b) 242, 2224 (2005).

24 J. Keeling and N.G. Berloff, Phys. Rev. Lett. 100, 250401 (2008).
25 M.O. Borgh, J. Keeling, and N.G. Berloff, Phys. Rev. B 81, 235302 (2010).

26 M.O. Borgh, G. Franchetti, J. Keeling, and N.G. Berloff, Phys. Rev. B 86, 035307 (2012).

27 G. Tosi G. Christmann, N.G. Berloff, P. Tsotsis, T. Gao, Z. Hatzopoulos, P.G. Savvidis, and J.J. Baumberg, Nature Phys. 8, 190 (2012).

28 D.J. Frantzeskakis, J. Phys. A 43, 213001 (2010).

29 G. Herring, L.D. Carr, R. Carretero-González, P.G. Kevrekidis, and D.J. Frantzeskakis, Phys. Rev. A 77, 023625 (2008).

30 T. Kapitula, P.G. Kevrekidis, and R. Carretero-González, Physica D 233, 112 (2007).

31 G. Theocharis, D.J. Frantzeskakis, P.G. Kevrekidis, B.A. Malomed, and Yu.S. Kivshar, Phys. Rev. Lett. 90, 120403 (2003); G. Theocharis, P. Schmelcher, M.K. Oberthaler, P.G. Kevrekidis, and D.J. Frantzeskakis, Phys. Rev. A 72, 023609 (2005).

32 K. Nozaki and N. Bekki, J. Phys. Soc. Jpn. 53, 1581 (1984); K. Nozaki and N. Bekki, Phys. Lett. A 110, 133 (1985).

33 M. Bazhenov and M. Rabinovich, Phys. Lett. A 179, 191 (1993). M. Bazhenov and M. Rabinovich, Physica D 73, 318 (1994).

34 It is especially interesting and relevant to note here that very soon after the posting of this manuscript (under index 1308.6798) in arXiv, a new experimental paper was posted under L. Dominici, D. Ballarini, M. De Giorgi, E. Cancellieri, B. Silva Fernández, A. Bramati, G. Gigli, F. Laussy and D. Sanvitto, arXiv:1309.3083. This manuscript principally focuses on the spontaneous emergence of ring dark solitons in recent state-of-the-art, highly nonlinear experiments in polariton superfluids; see especially Figs. 2-4 therein. This is a remarkable, independent verification of the robustness of the structures and of the principal associated predictions that are presented in this section. Notice that in that setting the formation of the waves is achieved by generating a drop of polariton condensate which was instantaneously created (and allowed to evolve) on the backdrop of a previously unperturbed state. This is sufficient to spontaneously give rise to the ring dark solitons (and to other remarkable features including shock waves etc.)

35 E.A. Ostrovskaya, J. Abdullaev, A.S. Desyatnikov, M.D. Fraser, and Yu.S. Kivshar, Phys. Rev. A 86, 013636 (2012).

36 C.J. Pethick and H. Smith, Bose-Einstein condensation in dilute gases (Cambridge University Press, Cambridge, 2002).

37 L.P. Pitaevskii and S. Stringari, Bose-Einstein Condensation (Oxford University Press, Oxford, 2003).

38 C.-K. Lam, B.A. Malomed, K.W. Chow, and P.K.A. Wai, Eur. Phys. J. Special Topics 173, 233 (2009); C.H. Tsang, B.A. Malomed, C.-K. Lam, and K.W. Chow, Eur. Phys. J. D 59, 81 (2010); F. Kh. Abdullaev, V.V. Konotop, M. Salerno, and A.V. Yulin, Phys. Rev. E 82, 056606 (2010); V. Skarka, N.B. Aleksić, H. Leblond, B.A. Malomed, and D. Mihalache, Phys. Rev. Lett. 105, 213901 (2010); Y.V. Kartashov, V.V. Konotop, and V.A. Vysloukh, Europhys. Lett. 91, 34003 (2010); M.J. Ablowitz, T.P. Horikis, S.D. Nixon, and D.J. Frantzeskakis, Opt. Lett. 36, 793 (2011); Y.V. Kartashov, V.V. Konotop, and V.A. Vysloukh, Opt. Lett. 36, 82 (2011); D.A. Zezyulin, Y.V. Kartashov, and V.V. Konotop, Opt. Lett. 36, 1200 (2011); M.J. Ablowitz, S.D. Nixon, T.P. Horikis, and D.J. Frantzeskakis, Proc. Roy. Soc. London A 467, 2597 (2011); 
Y.V. Kartashov, V.V. Konotop, and V.A. Vysloukh, Phys. Rev. A 83, 041806(R) (2011); M.J. Ablowitz, S.D. Nixon, T.P. Horikis, and D.J. Frantzeskakis, J. Phys. A 46, 095201 (2013).

39 B.A. Malomed, O. Dzyapko, V.E. Demidov, and S.O. Demokritov, Phys. Rev. B 81, 024418 (2010).

40 J. Cuevas, A.S. Rodrigues, R. Carretero-González, P.G. Kevrekidis, and D.J. Frantzeskakis, Phys. Rev. B 83, 245140 (2011).

41 The modification of the Newton-Raphson method consists in performing the (singular) Jacobian inversion by means of a LSQR method. The Newton-Raphson is a fairly standard method to solve boundary value problems which amount to systems of nonlinear equations (upon suitable discretization); see e.g. K.E. Atkinson, An Introduction to Numerical Analysis, (John Wiley and Sons, New York, 1989) for a detailed analysis of the method and its convergence properties, including its major advantage, namely the quadratic convergence, provided a sufficiently good initial guess. This is also the potential weakness of the method, namely an inefficient initial guess may not converge or converge to an entirely different solution. Here, we use the method along side parametric continuation (i.e., we find the solution for a set of parameters and then slightly modify the parameters using as initial guess for the Newton method the solution at the previous step/parameter value). It should also be noted that indeed for a single parameter value, multiple solutions (of different types may co-exist).
We will see multiple such examples below. In these cases, the Newton-Raphson method converges to these different solutions by virtue of the use of different initial seeds.

42 S. Middelkamp, P.G. Kevrekidis, D.J. Frantzeskakis, R. Carretero-González, and P. Schmelcher, Phys. Rev. A 82, 013646 (2010); see also R. Kollár and R.L. Pego, Appl. Math. Res. Express 1, 1 (2012).

43 V. Achilleos, P.G. Kevrekidis, D.J. Frantzeskakis, and R. Carretero-González, Phys. Rev. A 86, 013808 (2012).

44 D.A. Zezyulin and V.V. Konotop, Phys. Rev. A 85, 043840 (2012).

45 Movies for the dynamical evolution presented in Figs. 9 18 are presented as Supplemental material available at html://iop.org/aaaaaa. These movies may significantly facilitate the understanding of the unstable dynamical evolution for the reader.

46 O.V. Borovkova, V.E. Lobanov, Y.V. Kartashov, and L. Torner, Opt. Lett. 36, 1936 (2011).

47 R. Navarro, R. Carretero-González, P.J. Torres, P.G. Kevrekidis, D.J. Frantzeskakis, M.W. Ray, E. Altuntaş, and D.S. Hall, Phys. Rev. Lett. 110, 225301 (2013).

48 J.R. Anglin and M. Crescimanno, arXiv:condmat/0210063.

49 I. Coddington, P. Engels, V. Schweikhard, and E.A. Cornell, Phys. Rev. Lett. 91, 100402 (2003).

50 H. Pu, L.O. Baksmaty, S. Yi, and N.P. Bigelow, Phys. Rev. Lett. 94, 190401 (2005). 